\title{
CONSTRUCTION OF CONIC SECTIONS BY PAPER-FOLDING.
}

\section{By Alfred J. LotkA,}

Research Laboratory, Laurel Hill Works, L. I.

A method has been described* for constructing a parabola as the envelope of the creases formed on folding a piece of paper in such manner that a fixed point always falls upon a fixed straight line.

The other conic sections also can be similarly obtained, if for the straight line a circle is substituted ${ }^{* * *}$, as is shown by the ac-

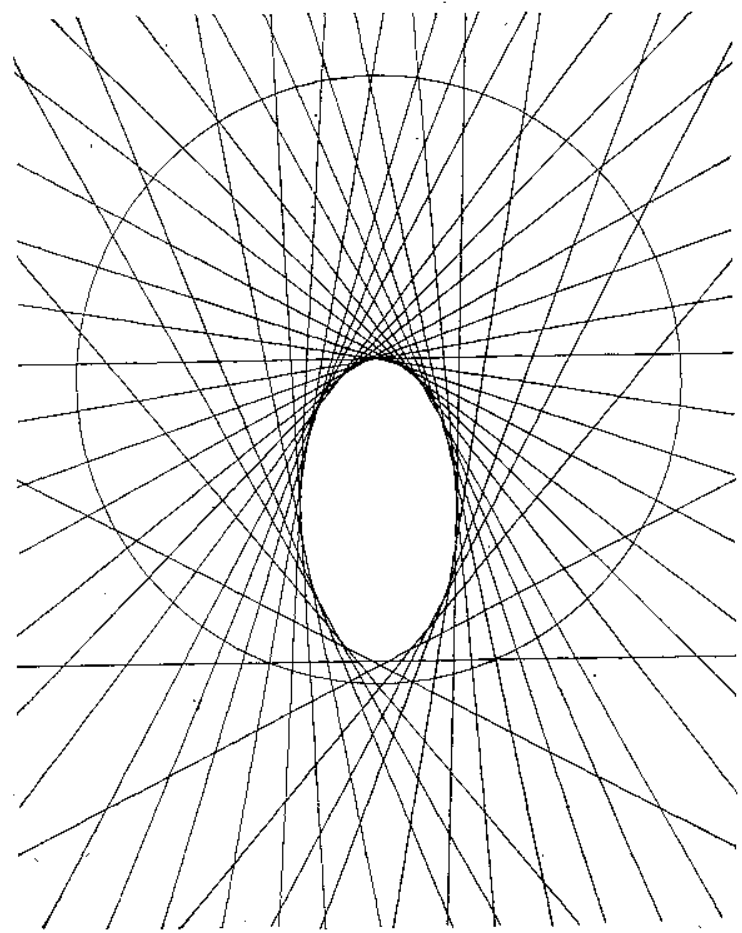

FlG.l.

companying examples (Figs. I and 2), and by the following ana1ytical demonstration.

Referring to Fig. 3, let $C$ be the center of the fixed circle, and $P$ the fixed point. p. 116.

*S. Row (W. W. Beman and D. E. Smith) Geometric Exercises in Paper-Folding, 1901,

**It is, of course, necessary to use translucent paper (tracing paper), or, if using opaque paper, to mark the fixed point on the back of the sheet, and on the edge of a perforation tnade in the same. 
Bisect $\mathrm{CP}$ in $\mathrm{O}$, and make $\mathrm{O}$ the origin of a system of rectangtular co-ordinates, with $\mathrm{OP}$ for $\mathrm{X}$ axis. Let $\mathrm{OP}=x_{0}$.

Then the paper is so folded that $P$ falls upon some point $Q$ of the circle.

If $x_{1} y_{1}$ are the co-ordinates of $Q$ we have:

$\left(x_{1}+x_{0}\right)^{2}+y_{1}^{2}=\mathrm{R}^{2} \ldots \ldots$ (1) where $R$ is the radius of the fixed circle.

If $x: y_{2}$ are the co-ordinates of the mid-point $\mathrm{S}$ of $\mathrm{PQ}$, then

$x_{1}=2 x_{2}-x_{0}$

$y_{1}=2 y_{2}$

Substituting these values in (I.) and simplifying:

$$
x_{2}^{2}+y_{2}^{2}=\frac{\mathrm{R}^{2}}{4}=r^{2} \text { say }
$$

i. e. the point $S$ lies upon a circle having its center at $O$, and a radius $r=\frac{R}{2}$

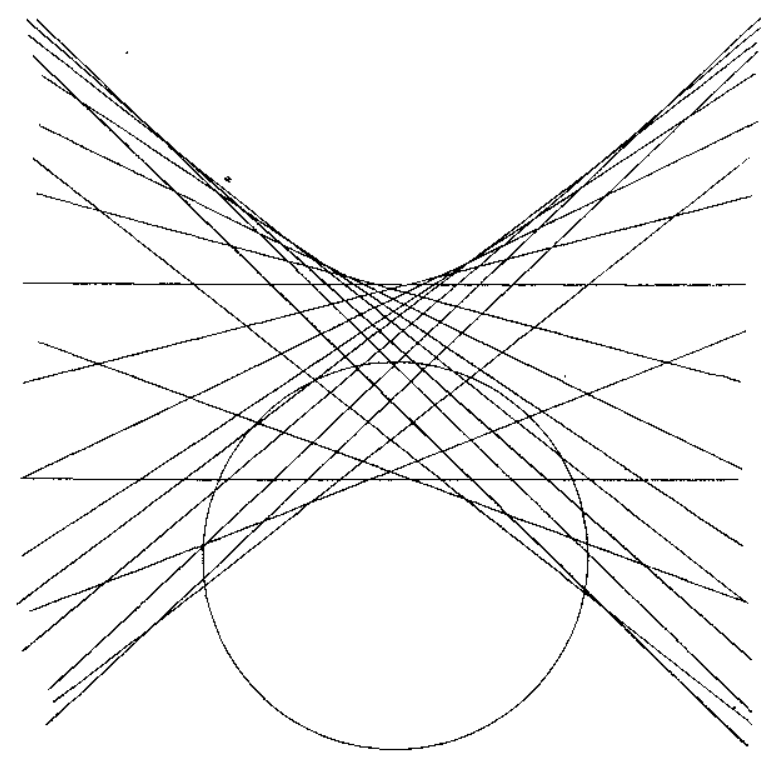

FIG.Z.

Now the crease produced is evidently RT, perpendicular to $\mathrm{PQ}$ in $\mathrm{S}$. Its equation is

$$
y-y_{2}=\frac{\left(x_{0}-x_{2}\right)}{y_{2}} \cdot\left(x-x_{2}\right)
$$


Rearranging and putting $r^{2}$ for $\left(x_{2}^{2}+y_{3}^{2}\right), x_{2}\left(x+x_{0}\right)=r^{2}+x x_{0}-$ $y y_{2}$.

Squaring $\quad x_{2}^{2}\left(x+x_{0}\right)^{2}=r^{4}+2 r^{2} x x_{0}+x^{2} x_{0}^{2}-2 y y_{2}\left(r^{2}+x x_{0}\right)+y^{2} y_{z}^{2}$ Putting $x_{2}^{2}=r^{2}-y_{2}^{2}$, simplifying, and arranging as a quadratic in $y_{2}$

$y_{2}^{2}\left\{y^{2}+\left(x+x_{0}\right)^{2}\right\}-2 y_{2} \gamma\left(r^{2}+x x_{0}\right)+r^{2}\left(r^{2}-x^{2}-x_{0}^{2}\right)+x^{2} x_{0}^{2}=0$

For a given pair of values of $x$ and $y$, equation (2), gives either two imaginary, two real and different, or two real and equal roots for $y^{2}$.

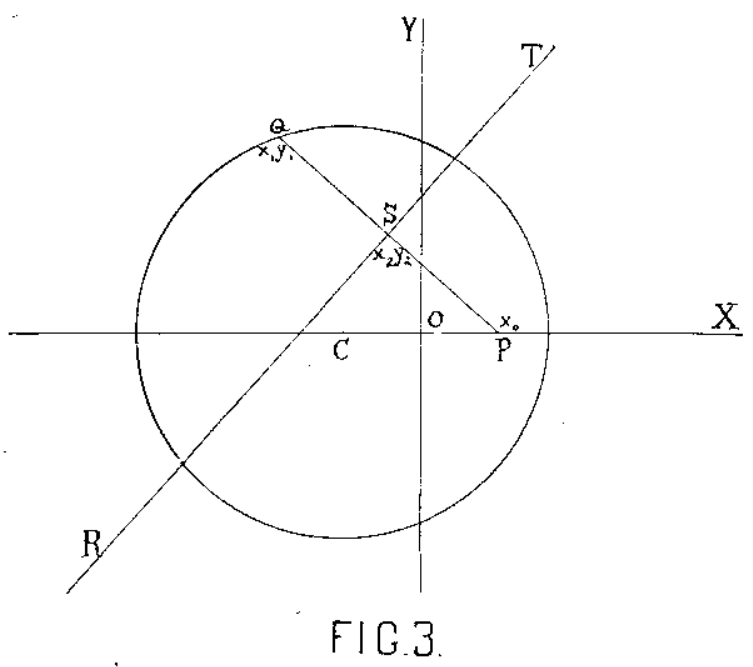

This means that the number of creases which can be produced through a given point in the manner specified is either 0,2 or $\mathrm{I}$.

The points through which only one crease can be made evidently lie on the envelope of the series of creases, the single crease through each being tangent at such point to the envelope, so that the condition for this envelope is given by the condition for the equality of the roots of (2), viz:

$$
\begin{gathered}
4 y^{2}\left(r^{2}+x x_{0}\right)^{2}-4\left\{y^{z}+\left(x+x_{0}\right)^{2}\right\}\left\{r^{2}\left(r^{2}-x^{2}-x_{0}^{2}\right)+x^{2} x_{\circ}^{2}\right\}=0 \\
r^{2} y^{2}+x^{2}\left(r^{2}-x_{0}^{2}\right)-r^{2}\left(r^{2}-x_{0}^{2}\right)=0 \\
\frac{x^{2}}{r^{2}}+\frac{y^{2}}{r^{2}-x_{0}^{2}}=1
\end{gathered}
$$

Hence the envelope is an ellipse or a hyperbola, according as $r>x_{0}$ or $r<x_{0}$, i. e., according as $\mathrm{P}$ lies within the fixed circle, or outside the same. 